\title{
Pontos de vista e conhecimentos dos sinais indicativos de depressão entre acadêmicos de enfermagem
}

\author{
UNDERGRADUATE NURSING STUDENTS' KNOWLEDGE AND VIEWPOINTS ON DEPRESSION \\ PUNTOS DE VISTA Y CONOCIMIENTOS DE LOS SIGNOS INDICATIVOS \\ DE DEPRESIÓN ENTRE ESTUDIANTES DE ENFERMERIA
}

\section{Antonia Regina Ferreira Furegato', Andréia Fernanda Nievas², Edilaine Cristina Silva ${ }^{3}$, Moacyr Lobo Costa $\mathrm{Jr}^{4}$}

\begin{abstract}
RESUMO
Objetivou-se identificar pontos de vista, conhecimento e sinais de depressão entre graduandos de Enfermagem. Quatro instrumentos foram utilizados para a coleta dos dados: A - Pontos de Vista Sobre Depressão; B - Conhecimento Sobre Depressão; C - Inventário de Beck; D - Escala de Zung. A análise quantitativa descritiva somou-se às reflexões qualitativas. Os alunos demonstraram que têm interesse e freqüentaram cursos de saúde mental; $65,4 \%$ tiveram altos escores de acordo com os pontos de vista $61,5 \%$ e têm ótimo conhecimento sobre depressão. Nas duas escalas de depressão verificou-se a existência de depressão moderada em um aluno. Nas respostas individuais, observa-se a falta de experiência dos alunos, preconceitos e informações equivocadas.
\end{abstract}

\section{DESCRITORES}

Depressão.

Saúde Mental.

Estudantes de enfermagem.

\begin{abstract}
This study aimed at identifying viewpoints, knowledge and indicative signs of depression among nursing students. Four instruments were used for data collection: A - Viewpoints on Depression; B - Knowledge on Depression; C - Beck's Inventory and D - Zung's Scale. The descriptive quantitative analysis added to qualitative reflections. Students showed that they were interested and that they had attended courses on mental health; $65.4 \%$ had high scores according to the expected viewpoints for depression; $61.5 \%$ showed excellent knowledge concerning depression. Two scales of depression showed moderate depression in one student. On basis of individuals responses, the students' lack of experience, some prejudice or erroneous information were observed.
\end{abstract}

\section{KEY WORDS}

Depressión.

Mental health.

Students, nursing.

\section{RESUMEN}

El presente estudio tuvo como objetivo identificar puntos de vista, conocimiento y signos de depresión entre estudiantes de Enfermería. Fueron utilizados cuatro instrumentos para la recolección de los datos: A Puntos de Vista Sobre Depresión; B - Conocimiento Sobre Depresión; C - Inventario de Beck; D Escala de Zung. El análisis cuantitativo descriptivo se sumó a las reflexiones cualitativas. Los alumnos demostraron que tienen interés y frecuentaron cursos de salud mental; $65.4 \%$ tuvieron altos escores de acuerdo con los puntos de vista esperados de depresión; $61.5 \%$ tienen óptimo conocimiento sobre el tema. En las dos escalas de depresión se verificó la existencia de depresión moderada en un alumno. En las respuestas individuales, se observa falta de experiencia de los alumnos, prejuicios e informaciones equivocadas.

\section{DESCRIPTORES}

Depresión.

Salud mental.

Estudiantes de enfermería.
1 Professora Titular do Departamento de Enfermagem Psiquiátrica e Ciências Humanas da Escola de Enfermagem da USP de Ribeirão Preto (EERP/USP).

furegato@eerp.usp.br

2 Mestranda da Enfermagem Psiquiátrica da EERP/USP.

3 Mestranda da Enfermagem Psiquiátrica da EERP/USP.

4 Professor Doutor do

Departamento de Enfermagem Psiquiátrica e Ciências Humanas da EERP/USP. 
Antonia Regina F. Furegato Andréia Fernanda Nievas Edilaine Cristina Silva Moacyr Lobo Costa Jr.

\section{INTRODUÇÃO}

Todas as pessoas passam por momentos nos quais se sentem tristes, sozinhas ou infelizes. Uma pessoa que sofreu perda de um ente querido ou perda do emprego pode sentir-se "deprimida". Na maioria das vezes, estará experimentando um sentimento normal e absolutamente compreensível.

O termo depressão, na linguagem corrente, pode designar um estado afetivo normal, um sintoma, uma síndrome ou várias doenças.

A depressão tem sido caracterizada como episódio patológico no qual existe perda de interesse ou prazer, distúrbios do sono e apetite, retardo motor, sentimentos de inutilidade ou culpa, distúrbios cognitivos, diminuição da energia e pensamentos de morte ou suicídio ${ }^{(1)}$.

Há relatos de que pacientes deprimidos que buscam atenção permanecem sem ser diagnosticados e sem receber tratamento, o que repercute em sofrimento, elevada morbidade e mortalidade além de contínuo risco de suicídio ${ }^{(2-3)}$. Além disso, por ser uma doença altamente incapacitante, interfere, na vida pessoal, profissional, social e econômica das pessoas acometidas.

A OPAS reconhece que os profissionais de saúde, nos diferentes níveis de atenção, não estão preparados para implementar ações que venham a preservar a saúde mental ou para diagnosticar a presença de transtornos mentais, na população ${ }^{(4)}$.

Implementando as atividades profissionais no cotidiano, presenciamos a falta de atendimento ao portador de depressão. Quando este é atendido, é acompanhado precariamente, não recebendo atenção adequada e eficaz, sendo considerado um chato, insistente, resistente.

A OPAS/OMS ${ }^{(4)}$ vem desenvolvendo programas educacionais para profissionais da saúde, capacitando-os para identificação e manejo dos transtornos afetivos. No Brasil, em 1998 começou a ser realizado um destes programas na UNICAMP junto aos médicos e, em 1999, através da EERP-USP junto a enfermeiros da Rede Básica de Saúde.

A humanização do cuidar se faz necessária. Conhecer o paciente, identificando suas necessidades é tarefa imprescindível para toda a enfermagem. $\mathrm{O}$ profissional deve estar alerta e ser sensível a pistas ocultas. É preciso uma atitude receptiva, disposição em escutar, observação acurada do comportamento e do conteúdo da comunicação do outro ${ }^{(5)}$.

Pelos motivos mencionados, educadores da enfermagem estão investindo na formação deste pro- fissional, para reconhecer e atuar terapeuticamente junto ao portador de transtornos afetivos.

\section{OBJETIVOS}

Identificar os pontos de vista, o conhecimento e sinais indicativos de depressão, entre acadêmicos de enfermagem.

\section{METODOLOGIA}

Com abordagem quantitativa, utilizamos a psicometria, ou seja, procedimentos estruturados e instrumentos formais para coletar a informação. Realizamos a análise qualitativa descritiva para os resultados individuais.

A medida escalar é uma das várias formas que a psicometria pode assumir, incluindo os testes psicológicos, os inventários, os questionários, as escalas, apresentando os parâmetros mínimos para se constituir em instrumento legítimo e válido ${ }^{(6)}$.

Esta pesquisa foi aprovada por Comitê de Ética em Pesquisa (EERP) e contou com o consentimento de cada um dos sujeitos.

\section{Local de estudo e População}

Fez parte do estudo um grupo de alunos do último ano do Curso de Graduação em Enfermagem da EERP/USP. Metade da turma (40), reunida para aula teórica foi convidada a participar, sendo explicados os objetivos e fornecidos os esclarecimentos devidos.

\section{Instrumentos e coleta dos dados}

Foram utilizados 4 instrumentos, testados e auto-aplicáveis:

A) Pontos de vista sobre depressão

B) Conhecimento do enfermeiro sobre depressão

C) Inventário de Beck e

D) Escala de Zung.

O instrumento A possibilita identificar as atitudes do sujeito com relação à depressão e o $\mathrm{B}$ pode avaliar os conhecimentos acerca da assistência de enfermagem à depressão, em nível de atenção primária. Estes dois instrumentos foram criados pela OPAS/OMS, para desenvolvimento de um programa educativo visando a redução e o controle dos transtornos afetivos nas Américas ${ }^{(4)}$.

A presença de sintomatologia depressiva entre os enfermeiros é um fator que poderá interferir no seu desempenho profissional. 
As escalas (C e D) permitem conhecer os sinais indicativos de depressão, fazer o diagnóstico, além do acompanhamento do paciente e o resultado dos tratamentos ${ }^{(7)}$. Foram aplicadas apenas com a finalidade de detectar e indicar a presença ou ausência de sintomatologia depressiva nos estudantes, os quais foram informados e orientados, nos casos positivos.

\section{Procedimento de análise dos dados}

Os resultados gerais dos instrumentos Ae B foram analisados estatisticamente. A reflexão sobre cada item, por conjunto das respostas permitiu verificar a opinião e o conhecimento do grupo sobre depressão.

Dos instrumentos C e D (Inventário de Beck e a Escala de Zung) apresentamos os resultados gerais, identificando os sinais de disforia e depressão nos grupos investigados, comparando os resultados.

\section{APRESENTAÇÃOE ANÁLISE DOS DADOS}

\section{A - Características dos sujeitos}

Dos 26 estudantes, a maioria (25) era do sexo feminino sendo 24 solteiros, com idade concentrada abaixo dos 25 anos (exceto 1 com 36 ).
Aproximadamente metade dos indivíduos $(57,7 \%)$ indicou alto interesse pela área de saúde mental e $42,3 \%$ dividiram-se entre médio e baixo interesse. Mais de dois terços $(69,2 \%)$ freqüentaram algum curso de saúde mental, nos últimos 3 anos.

A menção de alto interesse pela área é coerente com a informação sobre participação em cursos, inclusive entre os de médio e baixo interesse.

\section{B - Apresentação e discussão dos resultados dos Pontos de Vista sobre Depressão (A).}

A escala A identifica os pontos de vista do entrevistado sobre depressão. As 10 afirmativas oferecem a opção de respostas entre desacordo e acordo, numa graduação contínua de 10 opções com os escores: $\underline{\text { baixo }}(0$ a 2,9) $=$ desacordo; médio $(3,0$ a $6,0)=$ indeciso; alto $(6,1$ a 9,0$)=$ acordo. As questões 3,4,5,8 e 10 têm a afirmativa correta situada no desacordo. Para a análise estatística, foram invertidos seus valores.

Verificaram-se 17 acadêmicos $(65,4 \%)$ com escores entre 6,1 e 9,0. Portanto, seus pontos de vista estão de acordo com o resultado esperado para o teste. Não houve desacordo (Tabela 1).

Tabela 1 - Distribuição dos resultados gerais (escores) dos Pontos de Vista sobre Depressão, dos 26 graduandos de enfermagem. (Ribeirão Preto, SP)

\begin{tabular}{cccc}
\hline Escores & Pontos de Vista & Freqüência (No) & F reqüência (\%) \\
\hline 0 a 2,9 & Baixo & - & - \\
3,0 a 4,4 & Médio & - & - \\
4,5 a 6,0 & & 9 & 34,6 \\
6,1 a 7,4 & Alto & 16 & 61,6 \\
7,5 a 9,0 & & 1 & 3,8 \\
\hline Total & & 26 & 100,0
\end{tabular}

Este resultado é significativo, visto que os alunos do $4^{\circ}$ ano de Enfermagem cursaram recentemente as disciplinas de Saúde Mental e Enfermagem Psiquiátrica. O conhecimento aprendido deu-lhes a condição de ter opiniões atuais e de acordo com o que se espera de um profissional de saúde que vai cuidar da população afetada por problemas emocionais.

As respostas dos alunos de enfermagem às 10 questões estão distribuídas na Tabela 2, dentro das opções de respostas, variando do desacordo ao acordo. Em nenhuma das afirmativas foram usadas todas as opções de repostas.
Analisando os resultados, por questão, observa-se na Tabela 2 que a $n^{\circ} 2$ foi a que apresentou maior quantidade de desacordo. Treze dos 26 alunos $(50 \%)$ estão totalmente em desacordo com a afirmativa de que Deprimir-se é um modo utilizado pelas pessoas frágeis para enfrentarem as dificuldades da vida. Além disso, outros 10 alunos (38\%) estão indecisos. Estes resultados levam a questionar se os alunos entenderam bem a questão formulada ou se de fato eles pensam que a depressão não tem ligação com situações de fragilidade.
Pontos de vista e conhecimentos dos sinais indicativos de depressão entre acadêmicos de enfermagem 
Antonia Regina F. Furegato Andréia Fernanda Nievas Edilaine Cristina Silva Moacyr Lobo Costa Jr.

Tabela 2- Distribuição das respostas dos $\mathbf{2 6}$ graduandos de Enfermagem às 10 questões dos Pontos de Vista sobre Depressão. (Ribeirão Preto, SP)

\begin{tabular}{|c|c|c|c|c|c|c|c|c|c|c|c|c|c|c|}
\hline \multirow[b]{2}{*}{ Questões } & \multicolumn{4}{|c|}{ Desacordo } & \multicolumn{5}{|c|}{ Indeciso } & \multicolumn{4}{|c|}{ Acordo } & \multirow[b]{2}{*}{ Total } \\
\hline & 0 & 1 & 2 & Subtotal & 3 & 4 & 5 & 6 & Subtotal & 7 & 8 & 9 & Subtotal & \\
\hline 1. I dentificação de casos & & & & 0 & 1 & 1 & 9 & 6 & 17 & 6 & 3 & & 9 & 26 \\
\hline 2. Pessoas frágeis & 11 & 1 & 1 & 13 & 3 & 2 & 2 & 3 & 10 & 2 & 1 & & 3 & 26 \\
\hline 3. Irritantes * & 12 & 3 & 4 & 19 & 3 & 1 & 1 & & 5 & & 2 & & 2 & 26 \\
\hline 6. Atenção primária & & 1 & 1 & 2 & 1 & 1 & 5 & 7 & 14 & 1 & 4 & 5 & 10 & 26 \\
\hline 7. Risco de suicídio & & & & 0 & & 1 & & 4 & 5 & 8 & 6 & 7 & 21 & 26 \\
\hline 8. Nada faz melhorar* & 22 & 2 & 1 & 25 & & & & 1 & 1 & & & & 0 & 26 \\
\hline 9. Adesão aos tratamentos & 1 & & & 1 & 1 & 1 & 2 & 4 & 8 & 2 & 7 & 8 & 17 & 26 \\
\hline 10. Procura compaixão* & 8 & 3 & 3 & 14 & 3 & 3 & 2 & & 8 & 3 & & 1 & 4 & 26 \\
\hline
\end{tabular}

*Questões com resultado invertido

Se o enfermeiro não considera que a depressão tenha ligação com situações de fragilidade, que cuidado ele poderá prestar a essa pessoa, ou a que situação ele estará associando a depressão?

Entretanto, na questão ${ }^{\circ} 4$, mais da metade (14 alunos) discorda e 10 estão indecisos quanto a: $A$ depressão é a forma de ser de alguns pacientes, difícil de ser modificada, o que se contrapõe à questão anterior a respeito da fragilidade.

A depressão não é uma forma de ser da pessoa, é uma circunstância pela qual ela está passando, tornando-a frágil por uma falha no funcionamento biológico, psicológico e/ou social.

Na questão ${ }^{\circ} 5$, apenas um aluno concorda totalmente que Trabalhar com os pacientes deprimidos é uma tarefa agradável e $15(57,6 \%)$ estão indecisos, o que desperta atenção para a inex-periência destes alunos no cuidado aos portadores de depressão.

Quando comparamos esse resultado com a questão $\mathrm{n}^{\circ}$ 3, encontramos 19 alunos (73\%) que não se irritam com pacientes deprimidos, o que vem reforçar que eles estão respondendo com base no idealismo da enfermagem, porém sem vivência.

Quem tem experiência em cuidar de paciente portador de depressão ou que tenha este no seu convívio diário não daria essas respostas. Cuidar de paciente deprimido é uma tarefa cansativa, demandando muita dedicação do profissional porque é um paciente difícil no trato, demonstrando apatia e aparentando às vezes não reagir em benefício próprio ${ }^{(3)}$.

O portador de depressão em geral perdeu o interesse pelas atividades costumeiras, apresenta uma visão de si mesmo dependente da dos outros, humor depressivo, redução da capacidade de experimentar prazer, fadiga ao menor esforço, lentidão das ações, dificuldade de se concentrar e tomar decisões, alterações no sono, apetite e libido, retraimento social com choro fácil e pessimismo geral $^{(8-9)}$.

Na questão $\mathrm{n}^{\circ} 8$, os alunos discordam que Atender pacientes deprimidos é uma perda de tempo, já que nada os faz melhorar. $\mathrm{O}$ aluno que pensa desta forma vai trabalhar no sentido de ajudar essas pessoas, pois eles acreditam na recuperação. Desses 26 alunos, encontramos $21(80,7 \%)$ concordando que podem contribuir para diminuir o risco de suicídio nos pacientes deprimidos.

Entretanto, na questão $\mathrm{n}^{\circ}$ 6, 10 deles (38\%) concordam que Grande parte desses pacientes pode ser assistida em nível primário, demonstrando atitude favorável em relação ao atendimento dos mesmos. Entretanto, 14 (58,3\%) estão indecisos quanto a esta possibilidade.

A maioria dos pacientes deveria ser atendida em centros de nível primário de atenção tendo em vista a posição estratégica do pessoal de saúde para detectar e tratar prematuramente a enfermidade e suas conseqüências através de apoio, medidas preventivas, suporte familiar, manejo psicológico e farmacológico e/ou encaminhamento ao especialista $^{(4)}$.

Na questão ${ }^{\circ} 9$, observa-se que 17 estudantes (65\%) concordam que podem contribuir para melhorar a adesão dos pacientes ao tratamento com antidepressivos.

A Enfermagem tem um papel importante na assistência ao paciente deprimido não se resumindo apenas em contribuir com o tratamento medicamentoso, que é importantíssimo, visto que seus efeitos vão produzir a remissão dos sintomas e a melhora da qualidade de vida dessas pessoas. Mas não é só isso que a enfermagem pode fazer. Escutar 
o paciente, ajudá-lo a tomar consciência da situação e perceber seu papel na manutenção e na supressão de sintomas, mostrar que está ali para apoiálo e orientá-lo sempre que necessário são atitudes esperadas do enfermeiro.

Na questão $\mathrm{n}^{\circ}$ 10, 14 alunos $(53,2 \%)$ discordam e outros $8(30,7 \%)$ estão indecisos a respeito de que As pessoas deprimidas, em geral, querem compaixão, não procuram curar-se. Os alunos entendem que é a depressão manifestação de um estado emocional que se configura numa doença e como tal deve ser adequadamente tratada.

\section{C - Apresentação e discussão dos resultados do conhecimento sobre depressão(B)}

Os resultados gerais das respostas mostraram que a maioria $(61,5 \%)$ dos acadêmicos têm alto conhecimento sobre depressão, 34,6\% têm médio conhecimento e apenas um indivíduo demonstrou baixo conhecimento (Tabela 3 ).

Comparando esses resultados com uma pesquisa junto a 73 enfermeiros, com este mesmo instrumento $^{(3)}$, verifica-se que o conhecimento dos alunos enquadra-se em alto, enquanto que os enfermeiros encontram-se distribuídos em médio e baixo $(8,2 \%)$.

Tabela 3 - Distribuição de freqüência de acertos dos alunos a respeito do seu Conhecimento Sobre Depressão. (Ribeirão Preto, SP)

\begin{tabular}{cccc}
\hline Escores & Pontos de Vista & Freqüência (\%) & F reqüência (\%) \\
\hline 0 a 4 & Baixo & 1 & 3,8 \\
5 a 8 & Médio & 9 & 34,6 \\
9 a 12 & Alto & 16 & 61,5 \\
\hline Total & & 26 & 100,0 \\
\hline
\end{tabular}

Esperava-se que os alunos apresentassem alto conhecimento sobre depressão, tanto na quantidade como também na qualidade das respostas, tendo em vista que cursaram, recentemente, as disciplinas de Saúde Mental e Enfermagem Psiquiátrica.

As quatro questões com maior índice de acerto(1E, 3D, 6D, 9E e 11E) tinham como opção "todas as anteriores" para a resposta correta (Tabela 4). Entretanto, essas questões são referentes às características da depressão, situações associadas, sintomas que acompanham o humor depressivo, ou seja, são indicativos de que os alunos têm conhecimento adequado sobrenoções básicas de depressão.

Tabela 4 - Distribuição das respostas dos 26 graduandos de Enfermagem às 12 questões de Conhecimento sobre Depressão. (Ribeirão Preto, SP)

\begin{tabular}{|c|c|c|c|c|c|c|c|}
\hline Questões & A & B & C & $\mathrm{D}$ & $E$ & Sem resposta & Total \\
\hline 1. Epidemiologia & - & 1 & 3 & - & $22 *$ & - & 26 \\
\hline 2. Diagnóstico & - & $21^{*}$ & 5 & - & - & - & 26 \\
\hline 3. Conduta & 4 & 1 & - & 19* & 2 & - & 26 \\
\hline 4. Tratamentos & - & - & 1 & $13^{*}$ & 10 & 2 & 26 \\
\hline 5. Psicoterapias & - & 8 & $12 *$ & 4 & 2 & - & 26 \\
\hline 6. Situações associadas & 3 & 1 & - & $22 *$ & - & - & 26 \\
\hline 7. Critérios diagnósticos & 1 & - & - & $23 *$ & 2 & - & 26 \\
\hline 8. E feitos secundários & 8 & 1 & - & $15^{*}$ & - & 2 & 26 \\
\hline 9. Grupos de risco & 1 & - & 1 & 2 & $22 *$ & - & 26 \\
\hline 10. Sinais indicativos & 12 & - & - & - & $14^{*}$ & - & 26 \\
\hline 11. Outros sintomas & 1 & - & - & 1 & $24 *$ & - & 26 \\
\hline 12. Conduta enfermeiro & - & - & - & - & $26 *$ & - & 26 \\
\hline
\end{tabular}

O maior índice de acertos (12E) refere-se à conduta terapêutica da enfermagem. Mesmo não tendo como avaliar esta postura na prática, teoricamente os alunos estão preparados para lidar com estas questões.
A 2B, teve alto índice de acertos. O diagnóstico médico é prerrogativa do médico. Entretanto, é importante que o enfermeiro saiba que não basta a presença de um ou outro sintoma para que seja feito este diagnóstico. Caso contrário, o profissional
Pontos de vista e conhecimentos dos sinais indicativos de depressão entre acadêmicos de enfermagem 
Antonia Regina F. Furegato Andréia Fernanda Nievas Edilaine Cristina Silva Moacyr Lobo Costa Jr. passaria a enxergar a depressão em sintomas isolados. Além do mais, sabendo esses indicadores, o enfermeiro pode fazer os devidos encaminhamentos e orientar melhor o pessoal que o auxilia nos cuidados em seu serviço assim como os familiares ou cuidadores.

Entretanto, causou surpresa verificar que 19,2\% dos alunos identificam o caso apresentado neste teste (que tem todas as evidências de depressão), como esgotamento nervoso. Isto é resposta de leigo (2C).

No que se refere às psicoterapias, as respostas mostram o desconhecimento de grande parte dos alunos, visto que 8 deles $(30,7 \%)$ consideram a psicanálise como a terapia de escolha. Este resultado também foi encontrado nos enfermeiros da rede básica de saúde, em pesquisa realizada recentemente $^{(3)}$. A terapia cognitiva (5D) tem sido muito utilizada para este tipo de transtorno, devido à sua técnica de ação imediata ${ }^{(10)}$.

No tratamento medicamentoso, $50 \%$ dos entrevistados (13 alunos) indicariam um tricíclico para o tratamento. Entretanto, é preocupante o fato de que 10 alunos $(38,4 \%)$ usariam o recurso da hipermedicação atuando nos sintomas secundários. Esta conduta tem sido muito observada nos serviços onde o portador de depressão é medicado pelos seus sintomas isoladamente e não como um caso de depressão que é composto pelo conjunto desses sintomas.

Sobre os efeitos colaterais $\left(n^{\circ} 8\right)$ em pacientes que utilizam antidepressivos tricíclicos, 15 alunos (57,6\%) têm noção clara de todos os efeitos esperados. Entretanto, 42,3\% apresentam desconhecimento sendo que muitos deles $(30 \%)$ consideram a secura na boca como o único sintoma secundário ao uso do medicamento.

Na questão 3B observa-se que a maioria (73\%) informaria ao médico, perguntaria se têm idéias de suicídio e sobre o consumo de álcool. Entretanto, 4 alunos apenas informariam ao médico. Lamentavelmente, a conduta desses $15,3 \%$ indica que o aluno de enfermagem mantêm-se numa postura de subserviência ao médico e deixando de valorizar outras ações da enfermagem.

Ao identificar um paciente com depressão seria de se esperar que o graduando de enfermagem fizesse uma observação completa do quadro apresentado, atentando para a presença de idéias suicidas e uso de substâncias psicoativas, dando os cuidados necessários e/ou encaminhando para atendimento especializado.
Nas respostas individuais, denota-se falta de experiência dos alunos, resquícios de preconceitos, assim como informações equivocadas. Em vista desse fato, espera-se que a enfermagem seja despertada para o seu papel terapêutico que além de complementar, inclui toda a comunicação, observação, cuidados diretos, condutas de orientação e gerenciamento do serviço para atender às necessidades apresentadas pelo paciente nas 24 horas.

\section{D - Apresentação e discussão dos resultados gerais do Inventário de Beck e Escala de Zung}

Pelos dados das Tabelas 5 e 6 verifica-se que não há nenhum caso de depressão grave entre os acadêmicos de Enfermagem. Entretanto, 3 deles podem ter depressão moderada. Dentre os sintomas assinalados com maior freqüência destacamos: crises de choro, tristeza, auto-depreciação, inibição para o trabalho, distúrbios do sono, fadiga e irritabilidade.

Para o Inventário de Beck, consideramos os escores recomendados para populações que não apresentam avaliação nem diagnóstico de depressão (acima de 15 = disforia, acima de 30 = depressão). Após estudos para adaptar e validar esta escala para a língua portuguesa, concluiu-se que ela é útil também como instrumento de medida dos elementos constitutivos da depressão, em pessoas sem diagnóstico desta condição clínica ${ }^{(7)}$.

Tabela 5 - Distribuição dos resultados gerais do Inventário de Beck, aplicado aos graduandos de Enfermagem. (Ribeirão Preto, SP)

\begin{tabular}{ccc}
\hline Resultados & Freqüência & Percentagem(\%) \\
\hline 0 & 4 & 15,4 \\
2 & 4 & 15,4 \\
3 & 3 & 11,5 \\
4 & 4 & 15,4 \\
6 & 1 & 3,8 \\
7 & 2 & 7,7 \\
Subtotal & 18 & 69,2 \\
10 & 2 & 7,7 \\
11 & 1 & 3,8 \\
13 & 1 & 3,8 \\
14 & 1 & 3,8 \\
Subtotal & 5 & 19,1 \\
17 & 1 & 3,8 \\
23 & 1 & 3,8 \\
25 & 1 & 3,8 \\
Subtotal & 3 & 11,4 \\
\hline Total & 26 & 100,0 \\
\hline
\end{tabular}


Tabela 6 - Distribuição dos resultados gerais da Escala de Zung (D), aplicada aos graduandos de Enfermagem. (Ribeirão Preto, SP)

\begin{tabular}{ccc}
\hline Zung & Freqüência & Percentagem(\%) \\
\hline 22 & 1 & 4,0 \\
24 & 3 & 12,0 \\
25 & 1 & 4,0 \\
26 & 1 & 4,0 \\
27 & 1 & 4,0 \\
28 & 2 & 8,0 \\
30 & 1 & 4,0 \\
31 & 2 & 8,0 \\
32 & 1 & 4,0 \\
34 & 1 & 4,0 \\
35 & 1 & 4,0 \\
39 & 2 & 8,0 \\
42 & 2 & 8,0 \\
43 & 1 & 4,0 \\
45 & 1 & 4,0 \\
46 & 1 & 4,0 \\
50 & 1 & 4,0 \\
51 & 1 & 4,0 \\
Subtotal & 24 & 96,0 \\
61 & 1 & 4,0 \\
Subtotal & 1 & 4,0 \\
\hline Total & 25 & 100,0 \\
\hline
\end{tabular}

Nos resultados das respostas de 25 sujeitos à Escala de Zung (Tabela 6) verifica-se que 24 não têm depressão (96\%) e apenas 1 aluno apresenta escore indicativo de depressão moderada. Consideram-se os escores: abaixo de 59 = ausência de depressão, de 60 a 69 = depressão moderada; acima de $70=$ depressão grave.

Na Escala de Zung, composta por 20 afirmativas, há referência a episódios de choro durante a semana (24\%) e 1 acadêmico tem vontade de chorar todos os dias. Em 36\% das respostas verificam-se alterações nos hábitos alimentares, e $48 \%$ admitem ter problemas intestinais, com alguma freqüência. Alguns alunos assinalaram alterações no funcionamento da mente; entretanto, $56 \%$ raramente sentem isso. Há referências de cansaço, sem razão, em 38\% dos alunos. Além disso, $44 \%$ referem dificuldades para fazer as atividades costumeiras e $40 \%$ alguma inquietação.

Há referência de otimismo em $84 \%$ das respostas. A maioria concebe a vida como boa e satisfatória embora 2 alunos não pensem assim. Um aluno sente-se mais irritado do que de costume, todos os dias.

No que se refere ao sentimento de utilidade pessoal, $11 \%$ dos alunos referiram que raramente se sentem úteis e necessários, $40 \%$ referem sentimento de inutilidade em alguns momentos e $44 \%$ têm dificuldades em tomar decisões.
Apenas 11 alunos (44\%) desfrutam das coisas como de costume e $1 \%$ deles raramente desfrutam. Um aluno (justamente o que foi indicado nos 2 testes) refere sentir que as pessoas estariam melhor se ele não estivesse vivo. Este aluno precisa de orientação sobre os indicadores encontrados, precisaria de apoio e estímulo para manter a sua rede de relações e encaminhamento para atendimento especializado. O mesmo foi procurado e devidamente orientado.

Confrontando-se as duas escalas, percebe-se que os resultados são muito parecidos, indicando a possibilidade de existência de depressão moderada em 1 aluno e presença dos mesmos sintomas: alteração do sono, apetite e falta de interesse pela vida nas duas situações.

\section{CONCLUSÕES}

Através deste estudo percebe-se que os alunos do $4^{\circ}$ ano de enfermagem têm interesse pela área de saúde mental, têm opiniões atualizadas e bom conhecimento sobre depressão, embora ainda não tenham aplicado o conhecimento teórico na prática profissional.

Entretanto, alguns alunos identificaram a contribuição do enfermeiro apenas na melhoria da adesão do paciente ao tratamento medicamentoso. Isto se configura em uma postura de submissão não valorizando as demais atividades do enfermeiro.

Quantitativamente, as respostas configuram opiniões adequadas e bom conhecimento sobre depressão. Entretanto, nas respostas individuais percebem-se lacunas no conhecimento, provavelmente, relacionadas a falhas no ensino deste tema aos estudantes.

Foram detectados sinais indicativos de disforia e depressão moderada entre alguns alunos e aqueles com possível depressão foram procurados, orientados e encaminhados para atendimento. 
Antonia Regina F. Furegato Andréia Fernanda Nievas Edilaine Cristina Silva Moacyr Lobo Costa Jr.

\section{REFERÊNCIAS}

(1) Kaplan HI, Sadock BJ. Tratado de psiquiatria. Porto Alegre: Artmed; 1997.

(2) Montgomery S. Confrontando la depressión. Guía del médico. New York: Pfizer Internacional; 1997.

(3) Silva MCF, Furegato ARF, Costa Jr ML. Depressão: pontos de vista e conhecimento do enfermeiro da rede básica de saúde. Rev Lat Am Enferm 2003; 11(1): 7-13.

(4) Organización Panamericana de Salud / Organización Mundial de la Salud (OPAS/OMS). Programa de Salud Mental - División de Promoción de Salud. Modelo para la capacitación de la enfermera general en la identificación y manejo de los transtornos afectivos. Washington (DC): Generalista I; 1999.

(5) Furegato ARF. Relações interpessoais terapêuticas na enfermagem. Ribeirão Preto: Scala; 1999.
(6) Pasquali L. Teoria e métodos de medida em ciências do comportamento: teoria e aplicação. Brasília: UNB; 1996.

(7) Calil HM, Pires MLN. Aspectos gerais das escalas de avaliação da depressão. Rev Psiq Clín 1998; 25(5): 240-4.

(8) Dalgalarrondo P. Psicopatologia e semiologia dos transtornos mentais. Porto Alegre: Artes Médicas; 2000.

(9) Lafer B, Almeida OP, Fraguas Jr G, Miguel EC. Depressão no ciclo da vida. Porto Alegre: Artes Médicas; 2000.

(10) Beck AT, Rush JA, Shaw BF, Gary E. Terapia cognitiva da depressão. Porto Alegre: Artes Médicas; 1997. 